\title{
Identificación del estado de madurez de las frutas con redes neuronales artificiales, una revisión
}

\section{Fruit ripeness identification with artificial neural networks - A review}

Fecha recepción: 31 de julio de 2015

Gustavo Andrés Figueredo-Ávila ${ }^{1}$

Fecha aceptación: 12 de octubre de 2015

Javier Antonio Ballesteros-Ricaurte ${ }^{2}$

\section{Resumen}

La aplicación de las Redes Neuronales Artificiales (RNA) y de la visión artificial tiene cada vez más acogida en la industria de productos alimenticios; estas técnicas priorizan la clasificación, el reconocimiento de patrones y la predicción de las cosechas y de los cambios físicos de sus productos. En este artículo se define el concepto de red neuronal y se describen sus principales características y modelos, y, por otro lado, se define el concepto de procesamiento de imágenes digitales y las diferentes etapas que lo componen. Complementariamente, se exponen las generalidades de la inspección de frutas (enfocada en Colombia) y sus técnicas. Finalmente, se especifican diferentes trabajos en los que se aplicaron técnicas de RNA y visión artificial en el campo de los productos alimenticios, dispuestos por áreas de aplicación, y se identifica de manera concluyente el impacto que estas dos técnicas tienen para la clasificación, el reconocimiento de patrones y la predicción en el sector de productos alimenticios.

Palabras clave: inspección de alimentos; procesamiento de imágenes; reconocimiento de objetos; Redes Neuronales Artificiales (RNA).

\begin{abstract}
The application of Artificial Neural Networks (ANNs) and artificial vision has received more and more acceptance in the food industry. These techniques prioritize the classification, pattern recognition, and prediction of the harvests and physical changes in the products. In order to understand the impact of these techniques, this article defines the concept of neural network and describes its main characteristics and models; and, on the other hand, defines the concept of digital imagery processing and its different stages. Complementarily, this review presents an overview of fruit inspection (focused on Colombia) and its techniques; and specifies and orders by application area different works in which ANNs techniques

1 Universidad Pedagógica y Tecnológica de Colombia (Tunja, Colombia).gustavoandres.figueredo@uptc.edu.co.

2 M. Sc. Universidad Pedagógica y Tecnológica de Colombia (Tunja, Colombia). javier.ballesteros@uptc.edu.co.
\end{abstract}


and artificial vision have been applied in the food industry. Finally, the impact of both techniques in the classification, pattern recognition and prediction in alimentary products area is conclusively identified.

Keywords: Artificial Neural Networks (ANN); food inspection; image processing; recognition of objects. 


\section{Introducción}

El crecimiento de las Redes Neuronales Artificiales (RNA) o, en inglés, Artificial Neural Network (ANN) ha sido tan importante que, inclusive, han encontrado participación en el campo de los productos alimenticios; tratando de mejorar la calidad de los productos se ha incluido este sistema para realizar labores de inspección de alimentos, teniendo en cuenta las características físicas de estos, y de predicción de cosechas, con el fin de mejorar el estado en que se recogen los alimentos y de distribuirlos en el punto exacto donde se genera más ganancia y el alimento es aprovechable. La visión artificial o las técnicas de procesamiento de imágenes han estado, frecuentemente, asociadas a la aplicación de RNA, permitiendo definir la forma como debe ser adquirida la información y cómo debe procesarse y tratarse para entenderla en el entorno computacional.

En este artículo se exponen los conceptos básicos de las RNA y del procesamiento de imágenes digitales, la temática sobre la inspección de frutas en Colombia y la aplicación de las redes neuronales para la clasificación de las frutas y, finalmente, las conclusiones.

\section{Contextualización}

Para el entendimiento de la investigación realizada, es necesario la definición de unos conceptos y teorías preliminares, que son expuestos en la presente sección.

\section{Redes Neuronales Artificiales (RNA)}

Existen varias definiciones de redes neuronales que ayudan a entender qué son, cuáles son sus propósitos y el porqué de ellas. Se puede afirmar que una red neuronal es un sistema que se adapta a las exigencias del entorno, dado que puede combinar técnicas que procesan la información de manera paralela (1); la red neuronal está en posibilidad de realizar simultáneamente varios procesos y mostrar un adecuado comportamiento. Por consiguiente, la red neuronal conforma todo un modelo computacional que, por sus componentes, potencian la interconexión y facilitan la adaptación
(2); igualmente, su característica adaptativa en general y sus componentes proveen un conjunto de soluciones y validación de secuencias que, desde la perspectiva de reconocimiento de patrones, son una extensión de métodos clásicos estadísticos (3).

Por otro lado, las RNA se dirigen, aunque de manera artificial, a imitar el comportamiento de los entes biológicos; para ello, modelizan matemáticamente dicho comportamiento, caso el cerebro humano (4). En ese sentido, el proceso de información que incorpora el sistema de red neuronal asume características de funcionamiento comunes a las redes neuronales biológicas (5) y, por consiguiente, se denominan adaptables y, aun, especializadas a circunstancias que las afectan. En todo el funcionamiento y organización de la red, logran que en el proceso de información se haga uso de principios semejantes a la estructura del cerebro humano (6), con lo cual la comprensión de las redes neuronales, el potencial de su utilización y su aplicación inducen a que se convierta en algo indispensable para la investigación, la industria y otras múltiples actividades humanas.

Existen múltiples modelos de redes neuronales, que han surgido de acuerdo con los avances tecnológicos, permitiendo desarrollar sistemas más complejos, eficientes y efectivos. Entre los modelos más conocidos están el perceptrón simple, propuesto por Rosenblatt (7) en 1957, que se define como una unidad básica de inferencia en forma de discriminador lineal y puede ser usado como neurona dentro de un perceptrón u otro sistema de red neuronal más grande. En 1960, Bernard Widrow y Marcial Hoff propusieron el modelo ADALINE, que mejora considerablemente la salida en comparación con el perceptrón, perfeccionando el manejo de errores. En 1985, Werbos (8) desarrolló el back-propagation, que es un algoritmo de aprendizaje supervisado y que, mediante el uso de derivadas, reduce de mejor manera los errores en la salida.

El surgimiento de modelos de redes neuronales se ha dado, en gran medida, debido a las necesidades y problemas que han traído la evolución en tecnología, los nuevos planteamientos matemáticos y físicos y la insistencia de los seres humanos por 
entender la naturaleza. En la Tabla I se presenta un

resumen de los modelos más conocidos y sus características (9) en el campo de redes neuronales.

Tabla I. Modelos de Redes Neuronales.

\begin{tabular}{|c|c|c|c|}
\hline Nombre & Aplicaciones & Comentarios & Limitaciones \\
\hline $\begin{array}{l}\text { Teoría de } \\
\text { resonancia } \\
\text { adaptativa (ART) }\end{array}$ & $\begin{array}{l}\text { Reconocimiento de patro- } \\
\text { nes (radar, sonar, etc.) }\end{array}$ & Sofisticada, poco utilizada & $\begin{array}{l}\text { Sensible a la translación, } \\
\text { distorsión y escala }\end{array}$ \\
\hline Back Propagation & $\begin{array}{l}\text { Síntesis de voz desde tex- } \\
\text { to. Control de robots. Pre- } \\
\text { dicción, reconocimiento } \\
\text { de patrones }\end{array}$ & $\begin{array}{l}\text { La red más popular. Nume- } \\
\text { rosas aplicaciones con éxi- } \\
\text { to. Facilidad de aprendiza- } \\
\text { je. Potente }\end{array}$ & $\begin{array}{l}\text { Necesita mucho tiempo para } \\
\text { el aprendizaje y muchos } \\
\text { ejemplos }\end{array}$ \\
\hline $\begin{array}{l}\text { Máquinas de } \\
\text { Boltzman y Cau- } \\
\text { chy }\end{array}$ & $\begin{array}{l}\text { Reconocimiento de pa- } \\
\text { trones (imágenes, sonar y } \\
\text { radar). Optimización }\end{array}$ & $\begin{array}{l}\text { Redes simples. Capacidad } \\
\text { de representación óptima } \\
\text { de patrones }\end{array}$ & $\begin{array}{l}\text { La máquina de Boltzman ne- } \\
\text { cesita un tiempo muy largo } \\
\text { de aprendizaje }\end{array}$ \\
\hline $\begin{array}{l}\text { Counter- propa- } \\
\text { gation }\end{array}$ & $\begin{array}{l}\text { Comprensión de imáge- } \\
\text { nes }\end{array}$ & $\begin{array}{l}\text { Combinación de percep- } \\
\text { trón y TPM }\end{array}$ & $\begin{array}{lll}\text { Numerosas } & \text { neuronas y } \\
\text { conexiones } & & \\
\end{array}$ \\
\hline Hopfield & $\begin{array}{l}\text { Reconstrucción de patro- } \\
\text { nes y optimización }\end{array}$ & $\begin{array}{l}\text { Puede implementarse en } \\
\text { VLSI, fácil de conceptuali- } \\
\text { zar }\end{array}$ & Capacidad y estabilidad \\
\hline Perceptrón & $\begin{array}{l}\text { Reconocimiento de carac- } \\
\text { teres impresos }\end{array}$ & La red más antigua & $\begin{array}{l}\text { No puede reconocer } \\
\text { caracteres complejos }\end{array}$ \\
\hline $\begin{array}{l}\text { Self-Organi- } \\
\text { zing-Map (SOM) }\end{array}$ & $\begin{array}{l}\text { Reconocimiento de patro- } \\
\text { nes, codificación de da- } \\
\text { tos, optimización }\end{array}$ & $\begin{array}{l}\text { Realiza mapas de caracte- } \\
\text { rísticas comunes de los da- } \\
\text { tos aprendidos }\end{array}$ & $\begin{array}{l}\text { Requiere mucho entrena- } \\
\text { miento }\end{array}$ \\
\hline
\end{tabular}

Fuente: Adaptado de (9).

Una red neuronal, independientemente del modelo, consta de cuatro elementos básicos (10):

1. Un conjunto de conexiones, pesos o sinapsis que determinan el comportamiento de la neurona. Estas neuronas pueden ser excitadoras (positivo) o inhibidoras (negativo).

2. Un sumador, que se encarga de agregar todas las entradas multiplicadas por la respectiva sinapsis.

3. Una función de activación no lineal para limitar la amplitud de salida de la neurona.

4. Un umbral exterior que determina el nivel por encima del cual la neurona se activa.
Dichos elementos permiten a la red neuronal delinear una estructura clave, a partir de la cual manifiesta su comportamiento y potencia la amplitud de soluciones a las que se enfrenta, es decir, la no linealidad en la activación, la variedad de conexiones y la perfectibilidad del resultado garantizan su complejidad, pero, por lo mismo, la sencillez para adaptarse al cambio.

Las redes neuronales son usadas en diferentes áreas del conocimiento, esencialmente para abordar problemas que no son posibles resolver con métodos formales. En el reconocimiento de imágenes se han trabajado las redes neuronales, ya que permiten identificar un objeto sin haber definido reglas y patrones muy complejos, si no que mediante el adecuado entrenamiento de la red con 
datos e imágenes, esta es capaz de inferir los atributos principales de ese objeto.

Es importante mencionar que el entrenamiento de una red neuronal debe ser adecuado, en términos de la cantidad de datos que se le ingresan, ya que, si se entrena con demasiados datos, el sistema dejará de inferir y lo que hará será aprender, por lo que no podrá identificar los objetos, a no ser que sean "exactos" según sus datos de entrada.

\section{Procesamiento de imágenes digitales}

El uso de RNA para identificar patrones en objetos, usualmente conlleva un paso previo, y de gran importancia, que permita garantizar que los datos con los cuales la red neuronal será entrenada le proporcionen un funcionamiento y una entrega de resultados adecuados; este paso es el procesamiento de las imágenes digitales, que permite organizarlas, verificar algún tipo de anomalía y aplicar

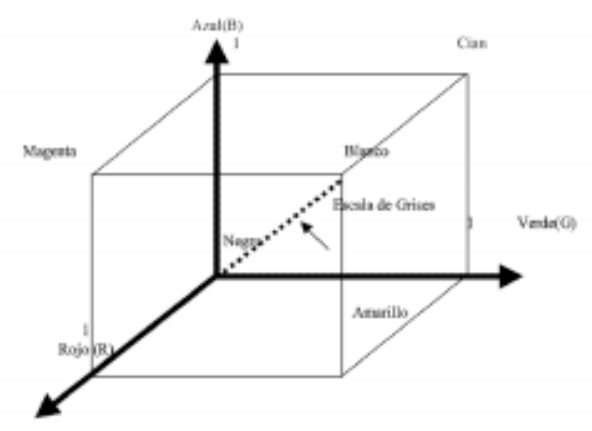

Figura 1. Cubo de color RGB (11).

Dentro de los modelos sensoriales también se encuentran el modelo CMY (Cian, Magenta, AmariIlo), que considera los colores secundarios de la luz, y el modelo YIQ, que separa la información de intensidad $(\mathrm{Y})$ de la información de color $(\mathrm{I}, \mathrm{Q})$ (11).

Por otro lado, los modelos perceptuales se enfocan en cómo se observan los objetos mediante la visión; entre estos modelos se encuentra el HSV (Hue, Saturation, Value, o Tono, Saturación, Va- técnicas que permitan mejorar la imagen original. El procesamiento de las imágenes digitales implica ejecutar una serie de pasos que garanticen modificar la imagen original y convertirla en aquella que resulte útil para su aplicación; estos pasos son:

A. Obtención de imágenes: Proceso que puede realizarse por medio de la utilización de un escáner o una cámara digital. Las imágenes son, comúnmente, digitalizadas en el formato RGB (Red, Green, Blue), en donde los valores posibles para cada entrada son de 255, representando el número de 8 bits, con una profundidad de 24 bits, lo que se representa en $\left(2^{8}\right)^{3}=16,77,216$ colores que se pueden formar con la combinación de estos tres colores primarios; este es un modelo sensorial, más orientado a los equipos electrónicos. Los colores se representan en coordenadas cartesianas, en donde cada color es un vector de origen y la diagonal principal corresponde a la escala de grises (Figura 1).

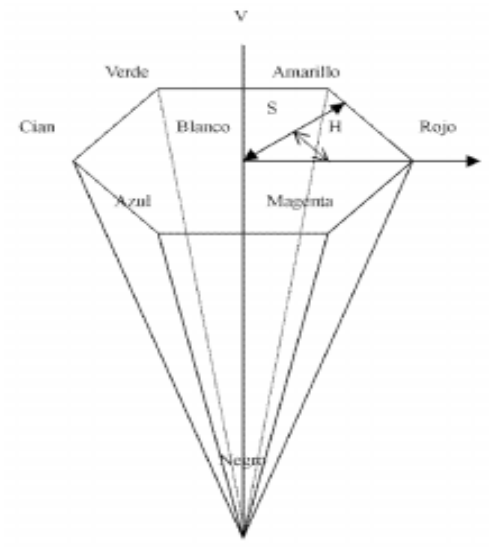

Figura 2. Modelo HSV (11).

lor), que se obtiene deformando el modelo RGB, de tal manera que se convierte en una pirámide hexagonal invertida, en donde el eje vertical es la brillantez o valor $(\mathrm{V})$; el eje horizontal, la saturación (S), y el ángulo de la proyección horizontal, el croma (H) (Figura 2). El HSL (Hue, Level, Saturation), otro modelo perceptual, se basa en coordenadas polares en 3 dimensiones, obteniendo un espacio en forma de dos conos unidos en una base (Figura 3) (11). 


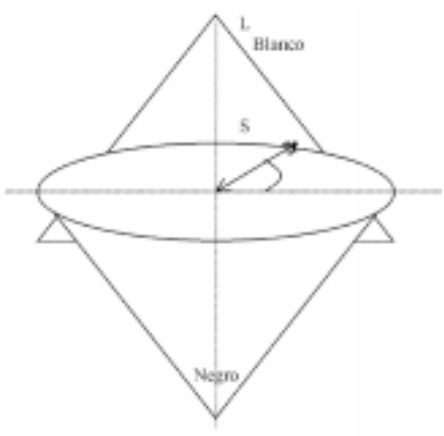

Figura 3. Modelo HSL (11).

El vértice inferior corresponde a negro; el superior, a blanco; el eje vertical representa la brillantez (L); el horizontal, la saturación (S), y el ángulo de la proyección horizontal, el croma $(\mathrm{H})$.

El modelo HSI (Tono, Saturación e Intensidad) separa el componente de intensidad de la información de color de una imagen (12); este se puede ver como una transformación del espacio RGB al espacio perceptual. El vértice inferior corresponde al negro, y el superior, al blanco; la intensidad (I) se mide en el eje vertical; la saturación (S), en función a la distancia a este eje, y el croma $(\mathrm{H})$ como el ángulo horizontal, tomado el rojo como referencia (cero grados) (11). En la Figura 4 se ilustra la distribución de cada componente.

En el momento de adquirir la imagen, es necesario considerar que hay ciertos factores que influyen en las características finales de esta; por ejemplo, la luz con la que se tome la imagen, la cantidad de pixeles, el tamaño y la distancia del objetivo a la cámara, entre otros.

B. Prepocesamiento: Se realizan acciones tales como reducción de ruido, realce de contraste, suavizado y operaciones aritméticas, con el fin de obtener características como las orillas, el color, la textura y el gradiente. Dentro de las principales acciones se encuentran:

1) Suavizado-Reducción de ruido: Las imágenes, usualmente, tienen cierta cantidad de ruido que puede afectar su calidad. El ruido puede clasificarse en:

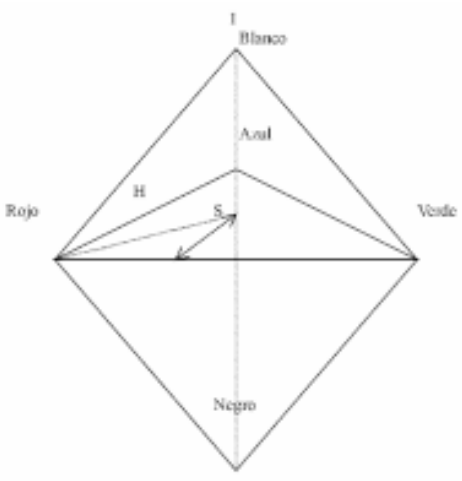

Figura 4. Modelo HSI (11).

a. Gaussiano: Se presentan variaciones en la imagen causadas por ganancias en la cámara, ruidos en digitalizadores o perturbaciones en la transmisión.

b. Impulsional: El valor del pixel de la imagen tiene relación con el valor del ruido, que toma valores muy bajos o muy altos.

c. Multiplicativo: Multiplicación de dos señales.

Entre las técnicas más usadas para la reducción del ruido se encuentran:

a. Media aritmética: Esta técnica busca ajustar el valor de los pixeles vecinos, tomando como referencia el valor promedio de los pixeles en una región (matriz de $3 \times 3$ ). Debido a esta característica, no se usa para la reducción del ruido en los bordes de la imagen.

b. Media ponderada: Consiste en modificar arbitrariamente el peso que tiene el pixel central en una matriz de $3 \times 3$, obligando a cambiar los pesos de los pixeles cercanos, para asegurar que la salida no sobrepase el valor del rango (la suma de los coeficientes de la matriz es la unidad).

c. Media Gaussiana: Se considera una curva gaussiana de revolución alrededor del pixel central de una matriz de convulsión.

d. Mediana: Normalmente se usa para reducir ruido de "sal" y "pimienta"; consiste en bus- 
car dentro de un conjunto de pixeles $(3 \times 3)$, un pixel que no sea completamente negro o completamente blanco, el cual se va a convertir en la salida de aquellos pixeles que en esa región sí son negros o blancos ( $\left.\begin{array}{lll}0 & 0 & 1\end{array}\right)$.

2) Realce de contraste: El contraste en una imagen existe, o es alto, si hay una diferencia entre los tonos que la componen, y es bajo si es difícil distinguir entre los elementos que la componen.

El histograma de una imagen permite contar la cantidad de pixeles presentes en cada canal; ejemplo, el modelo RGB presenta tres canales, y una imagen en tono de grises solo presenta uno. De aquí que la modificación del histograma de una imagen permite ajustar el valor del contraste. Este proceso de modificación se conoce como "Ecualización por histograma".

La Ecualización por Histograma trabaja sobre el nivel de intensidad dentro de la imagen, es decir, da un estimado de la probabilidad de ocurrencia de cada nivel de gris (11), por lo que es fácilmente aplicable a imágenes en tono de grises, pero si se aplica a una imagen en color bajo el modelo RGB modifica el color, a la vez que modifica el contraste, por lo que generalmente se aplica sobre la imagen con el modelo HSI, logrando que únicamente se modifique el histograma del canal Intensidad (I), y los otros dos componentes no se alteran.

C. Segmentación: Proceso de identificar los objetos presentes en una imagen, separándolos por regiones. Algunas de las técnicas más usadas para realizar este proceso se presentan en la Tabla II:

Tabla II. Técnicas de segmentación de imágenes.

\begin{tabular}{|l|l|l|}
\hline \multicolumn{2}{|c|}{ Técnica } & \multicolumn{1}{c|}{ Descripción } \\
\hline \multicolumn{2}{|c|}{ Detección de bordes } & $\begin{array}{l}\text { La técnica de detección de bordes busca diferenciar el objeto de interés de la imagen } \\
\text { de fondo, localizando los pixeles del borde. }\end{array}$ \\
\hline$\bullet \quad$ Canny & $\begin{array}{l}\text { Este modelo especifica que la imagen inicialmente se convulsiona con una función } \\
\text { gaussiana de dos dimensiones, para posteriormente computar el laplaciano de la ima- } \\
\text { gen convulsionada, y así poder hallar los pixeles de los bordes (13). }\end{array}$ \\
\hline$\bullet \quad$ Shen-Castan (ISEF) & $\begin{array}{l}\text { El algoritmo de Canny considera la obtención de todos los bordes sin omisión alguna, } \\
\text { localizándolos con la menor distancia posible entre los objetos que forman el borde } \\
\text { y evitando identificar múltiples pixeles en el borde cuando solo exista un borde. Este } \\
\text { algoritmo proporciona una reducción en el ruido, por lo que la imagen resultante suele } \\
\text { verse más borrosa que la original. }\end{array}$ \\
\hline$\bullet \quad$ Laplace & $\begin{array}{l}\text { A diferencia del algoritmo Canny, que aproxima un filtro óptimo por la derivada gaus- } \\
\text { siana, el algoritmo de Shen-Castan, usa un filtro óptimo directamente, acelerando el } \\
\text { proceso de búsqueda. }\end{array}$ \\
\hline$\bullet$ & $\begin{array}{l}\text { Este modelo se basa en las segundas derivadas o laplaciano de una Gaussiana (LoG), } \\
\text { logrando que se pueda estimar con mayor precisión la localización de la orilla (11). En } \\
\text { algunos casos es posible que, por la aplicación de las segundas derivadas, se presente } \\
\text { la aparición de dobles orillas. }\end{array}$ \\
\hline $\begin{array}{l}\text { Este modelo no solo permite detectar las orillas o bordes en una imagen, sino que a la } \\
\text { vez aplica suavizamiento para que la existencia de ruido no obstruya la detección de } \\
\text { los bordes. }\end{array}$ \\
\hline
\end{tabular}




\begin{tabular}{|l|l|}
\hline \multicolumn{1}{|c|}{ Técnica } & \multicolumn{1}{c|}{ Descripción } \\
\hline Segmentación por histograma & $\begin{array}{l}\text { Esta técnica asume que existe un solo objeto sobre un fondo uniforme, por lo que en } \\
\text { ese caso el histograma mostraría dos picos separados por un valle, el cual representaría } \\
\text { la separación entre las dos regiones. Usualmente, esta técnica presenta varios incon- } \\
\text { venientes, tal como: identificar las variaciones en el histograma cuando el valle no es } \\
\text { muy notorio y cuando las regiones varían suavemente su nivel, y se aplica solo cuando } \\
\text { existen pocas regiones. }\end{array}$ \\
\hline $\begin{array}{l}\text { Segmentación por crecimiento } \\
\text { de regiones }\end{array}$ & $\begin{array}{l}\text { Consiste en seleccionar puntos iniciales en las imágenes (pixeles), y a partir de estos } \\
\text { crecer paulatinamente por los pixeles similares hasta cierto límite. }\end{array}$ \\
\hline pegmentación por división-agru- & $\begin{array}{l}\text { Esta técnica hace uso de agrupamiento de regiones por métodos basados en pirámide o } \\
\text { en árboles cuaternarios, dividiendo las regiones en 4 y evaluando si satisface la medida } \\
\text { para determinar si se sigue dividiendo cada región en más partes. }\end{array}$ \\
\hline Semántica del dominio & $\begin{array}{l}\text { Esta técnica busca encontrar y separar las regiones teniendo cierta información a priori } \\
\text { que le permita enfocarse en cierta región de interés. Esta información a priori puede } \\
\text { ser dimensiones de la región, forma de la región, posición de la región en la imagen, } \\
\text { posición de la región respecto a otras regiones, etc. (11). }\end{array}$ \\
\hline
\end{tabular}

D. Representación y descripción: La imagen puede ser representada por una matriz binaria, que se compone únicamente de ceros y unos; por árboles binarios o cuaternarios, que expresan una jerarquía y organización de los valores que representan la imagen; por matriz de enteros, con los que se puede representar alguna característica propia de la imagen mediante un valor, ejemplo la intensidad. Por otro lado, una imagen ya segmentada puede ser descrita por un descriptor topológico, estadístico o geométrico.

\section{Resultados y discusión}

A continuación, se nombran algunos trabajos en los que se ha propuesto el uso de las RNA en el campo de los productos alimenticios.

A. Clasificación. En la clasificación se ha implementado la red neuronal en alimentos, como mandarinas, dátiles y manzanas, e, igualmente, se ha dado una visión general sobre productos alimenticios.

1) El color como principal característica para la inspección de alimentos. Como primera característica para la evaluación de la calidad en los alimentos se encuentra el color; por ejemplo, Ben-Hanan et al. (14) desarrollaron un sistema basado en redes neuronales artificiales de tipo BPN (Boltzman Perceptrón Neural Network) para clasificar las manzanas por color (entre rojas y verdes), considerando que este tipo de sistema acepta datos difusos y reentrenamiento basado en los datos a ser analizados, por lo que los resultados Ilegan a ser aproximados a lo que es la decisión de los expertos clasificadores de manzanas.

La consideración del color de la superficie de la manzana no es en sí la única condición que afecta la selección de su calidad; Yang (15) toma en cuenta tres características principales de la superficie de la manzana: el área no defectuosa, manchas como parches y manchas alargadas, datos para entrenar la red neuronal. El estudio se hizo con manzanas defectuosas, la precisión de la clasificación de estas manzanas fue del $96.6 \%$.

A su vez, en el afán de automatizar procesos manuales, y considerar de manera más general la superficie de la manzana, Nakano (16) propone como experimento usar dos redes neuronales artificiales para sustituir la inspección visual del trabajador: una identifica cualquier parte de la superficie de la manzana dentro de las características de "rojo normal", "rojo con magulladuras", "color rojo pobre", "color vino tinto" y "mucho o poco color de fondo", y la otra considera el color sobre toda la superficie de la manzana, y evalúa las características de "Superior", "Excelente", "Bueno", "Color pobre" y "Con magulladuras". 
2) Desarrollo de la topología de red. No solo el conjunto de datos de entrada va de alguna manera a proporcionar la salida correcta, en la mayoría de los casos, la topología en que se conforma la red neuronal proporciona una garantía de los resultados; así, con el fin de mejorar el proceso de ejecución de una RNA, Morimoto (17) hace uso de una red neuronal de múltiples capas y retroalimentación cuya topología fue diseñada por un algoritmo genético, logrando así un $73 \%$ de éxito en la clasificación.

La exactitud en el reconocimiento de los patrones depende, muchas veces, de la forma como es entrenada la red neuronal; Effendi (18) desarrolla una red neuronal artificial de propagación hacia atrás para identificar la maduración de la fruta Jatropha curcas y clasificarla dentro de una categoría de calidad, otorgando resultados con una exactitud del $95 \%$, con base en ejemplos de 27 imágenes.

El entrenamiento de las RNA depende en gran medida del problema que se va a atacar; así, si el objeto de estudio es diferente, la configuración de la red neuronal deberá ser diferente; para entender este punto se tiene que Mustafa et al. (19) utilizaron RNA para clasificar cinco frutas (manzanas, bananas, zanahorias, mangos y naranjas), tomando 17 características físicas para la evaluación, recolectando las imágenes de cámara digital ordinaria y procesando la información con el software Simulink de Matlab.

3) Las RNA como alternativas de solución. Debido a su flexibilidad y características de aprendizaje mencionadas, las redes neuronales fueron planteadas en algunos casos no solo como una nueva propuesta a la resolución de un problema, sino como alternativas a métodos ya existentes, así, Jayas et al. (20) hablan acerca del uso de redes neuronales como una alternativa a los modelos de regresión para la clasificación de productos agrícolas, en donde se le da importancia a la capacidad de adaptabilidad, procesamiento paralelo y tolerancia a fallos de las redes.
Por consiguiente, también Leemans et al. (21) realizan la comparación de dos métodos de clasificación: un análisis discriminante cuadrático y una RNA con un perceptrón multicapa y una capa oculta; esto para clasificar las manzanas en tres categorías: Extra I, II y rechazado.

La comparación de técnicas para clasificar los alimentos proporciona, muchas veces, información sobre en qué casos unas son eficientes y en qué casos no; Kadvir y Guyer (22) realizan la clasificación de las manzanas Empire y Golden Delicious haciendo uso de una red neuronal artificial con retropropagación y un clasificador estadístico como un árbol de decisión, identificando entre las manzanas en buen estado y mal estado; determinan que el clasificador estadístico es más productivo cuando el contraste entre las áreas saludables y dañadas en la superficie de la manzana es alto, mientras que la red neuronal es capaz de distinguir esa diferencia aun si ese contraste es débil.

Con el fin de aportar más a la caracterización de la RNA, Xiaobo et al. (23) a laboratory machine vision system was developed, which consisted of a color CCD camera equipped with an image grab device, a bi-cone roller device controlled by a stepping motor, and a lighting source. Four images, one for every rotation of $90^{\circ}$, were taken from each apple. Seventeen color feature parameters (FP) entrenan una red neuronal artificial con 20 nodos en la capa oculta, 200 ejemplos, unas 20.000 veces, con el fin de compararla con el método de parámetros de características de organización (OFP), concluyendo que para este caso el uso de redes neuronales no es el mejor, debido a su problema de generalización.

Esto hace pensar en la importancia de entrenar correctamente la red neuronal artificial, para que esta pueda inferir, mas no aprender, dado que la esencia de las RNA es la capacidad de mostrar resultados óptimos basados en un conjunto de datos de ejemplo que de alguna forma muestran el patrón a seguir.

4) Características que complementan a la del color. El crecimiento en la aplicación de las RNA es tal, que ya no solo se busca presentar- 
las como una alternativa de clasificación, sino, además, como un modelo capaz de evaluar sustancialmente más características a partir de un conjunto más cerrado; por ejemplo, Kondo et al. (24) hacen uso de redes neuronales artificiales para predecir el contenido de azúcar de la naranja a partir de la apariencia, y así clasificarla según su calidad.

Por otro lado, algunos apuntan a la percepción no solo del color, sino también de la forma, es decir, que la amalgama de características por evaluar aumenta el número de datos $y$, por ende, afecta la topología de la red; de este modo, Morimoto et al. (25) evalúan cuantitativamente el cambio de la forma de la fruta haciendo uso de RNA, que permite obtener una clasificación más sofisticada.

Respecto a los dátiles, Hobani, Thottam y Ahmed (26) hablan de clasificar 7 variedades de dátiles usando modelos incorporados, seleccionando atributos físicos y de color para cada variedad. Las principales características por medir en los dátiles son la mayor longitud, el mayor diámetro y dos diámetros menores. La estructura básica de la red neuronal es un modelo generado a partir del perceptrón multicapa y de back propagation. Se concluye que el clasificador de red neuronal perceptrón multicapa es mejor que un clasificador estadístico.

El color como evaluación de calidad de las frutas es solo una característica, a la que se le pueden sumar otras, como lo plantean Ashok y Vinod (27); estos proponen el uso de un clasificador de red neuronal probabilístico, al cual se le ingresan como datos de entrada los atributos externos de la manzana: el color, el tamaño, la forma, la textura y la presencia de daños. Consideraron Ashok y Vinod 20 imágenes del color de la manzana sin daños y 45 imágenes de la manzana con daños; la red neuronal fue capaz de distinguir el cambio en las características de la fruta con una exactitud de $86,52 \%$ y $88.33 \%$.

5) La importancia de los algoritmos de entrenamiento. Ya con los avances en computación y los nuevos algoritmos de entrenamiento, las redes neuronales toman mayor participación, con el fin de mejorar su eficiencia a la hora de reconocer patrones; entonces, ya no se deja únicamente la responsabilidad a los datos de entrada, y tampoco a la topología de la red, sino que también se entiende la importancia del algoritmo de entrenamiento de la red; así, un caso básico lo plantea Simões et al. (28), que proponen el uso de redes neuronales artificiales MLP con el algoritmo de retropropagación para la clasificación de frutas por el color de su superficie. Mientras tanto, como propuesta de mejora a lo que en sí ya hacen las redes neuronales, Ji y Yuan (29) consideran el uso de una red neuronal artificial entrenada con un algoritmo de optimización de enjambre de partículas (PSO) para clasificar las manzanas según su color, logrando un $94 \%$ de acierto en la clasificación.

Unay y Gosselin (30) realizaron pruebas de clasificación de la manzana "Jonagold" con diez clasificadores supervisados, entre los que se encuentran el perceptrón multicapa (MLP), la red neuronal de cascada hacia delante (CFNN) y la red neuronal Elman (ENN). Todas las redes neuronales usadas constaban de una arquitectura de dos capas con cinco neuronas en la capa oculta.

Para la clasificación de patrones existen multitud de algoritmos, así, por ejemplo, Zhang et al. (31) proponen el uso de un algoritmo de colonia de abejas artificial de aptitud y escalado (fitness-scaled chaotic artificial bee colony -FSCABC-), y una red neuronal hacia delante, para ser entrenada por un conjunto de imágenes tratadas previamente mediante el algoritmo de Split and Merge, las cuales contienen la información de la textura y la forma de las frutas.

6) Reconocimiento y tratamiento de imágenes. Es importante conocer que aunque, en muchos casos, el reconocimiento de las imágenes y su pretratamiento antes de ser reconocidas como datos de entrada para la red neuronal, son imágenes sencillas de alimentos; Brosnan y Sun (32), hablan del análisis de los alimentos, enfatizando en frutas, vegetales, granos y otro tipo de comida, utilizando la visión computacional para identificar $y$, posteriormente, de- 
tectar imperfecciones en la superficie de estos, mencionando los principales atributos de cada alimento; en conclusión, mencionan que los sistemas de visión computacional han venido siendo más utilizados en la industria alimenticia, pero aún existen ciertas dificultades a la hora de implementar este tipo de sistemas.

Así, para clasificar los gajos de mandarina, Fernández et al. (33), utilizando el concepto de firma del objeto (expresión que representa la distancia de cada punto de su perímetro al centro de masa como una función del ángulo) como generador de características, hacen una inspección visual de ellos y determinan que se obtienen mejores resultados que con el método manual; por lo cual, esta forma automatizada es adecuada para la inspección en línea.

Igualmente, Kavdir y Guyer (34) acompañan la red neuronal con imagen espectral, y destacan el impacto del manejo en la resolución de las imágenes respecto al rendimiento de la clasificación de las manzanas con el clasificador de red neuronal back propagation. De la misma manera, Shahin et al. (35) usan para ello redes neuronales y procesamiento de imágenes, y con adaptación para continuar la clasificación a pesar de la ocurrencia de posibles cambios en la superficie de las manzanas; el trabajo compara un clasificador neuronal con la técnica de análisis discriminante lineal para medir la efectividad en la clasificación de las viejas y nuevas magulladuras en dos tipos de manzanas.

7) Uso de RNA con otras técnicas para el reconocimiento de imágenes. Algunas veces, el acompañamiento de otras técnicas con las RNA puede proporcionar buenos resultados en la clasificación de las frutas; es así como Li y Zhu (36) proponen el uso de una red neuronal de back propagation y la teoría evidencial D-S para mejorar la exactitud en la selección de las manzanas, considerando las características de tamaño, forma y color extraídas de las imágenes procesadas de las manzanas.

Por su parte, Kurtulmus et al. (37) hacen uso de clasificadores estadísticos y redes neuronales artificiales, y soportado por un clasificador de vector para detectar la fruta del durazno en estado natural y bajo condiciones del entorno variantes, como son la iluminación del día; esto con el fin de identificar el estado de madurez del durazno y así evitar cosechar antes de su estado óptimo de recolección.

Shuihua et al. (38) proponen un sistema que consiste en wavelet entropy (WE), un componente principal de análisis (PCA), red neuronal artificial feedfordward (FNN) entrenada por el algoritmo de colonia de abejas artificial de aptitud y escalado (fitness-scaled chaotic artificial bee colony -FSCABC-) y optimización basada en biogeografía (BBO); evalúan 12 características de la fruta para realizar la clasificación por dos métodos diferentes, que consisten en el uso conjunto de $\mathrm{WE}+\mathrm{P}$ $\mathrm{CA}+\mathrm{FSCABC}-\mathrm{FNN}$ y WE$+\mathrm{PCA}+\mathrm{BBO}-\mathrm{FNN}$, obteniendo una exactitud con ambas combinaciones del $89.5 \%$.

B. Optimización. La optimización de los procesos para inspeccionar los alimentos también es un punto clave para utilizar las RNA, pues estas han demostrado poder reemplazar sistemas automatizados que alguna vez se encargaban de la clasificación e inspección de alimentos.

La red neuronal se muestra como una alternativa para resolver muchos problemas, $y$, en multitud de casos, resulta como alternativa exitosa; Morimoto et al. (39), usando una técnica de control inteligente de redes neuronales y algoritmos genéticos, proponen estudiar un patrón óptimo para tratar el calor en los tomates, teniendo en consideración el color de la superficie; la red neuronal consta de tres capas, como respuesta efectiva para identificar los cambios en el color del tomate; concluye que el tratamiento del calor puede no ser constante y cambia con el tiempo, el lugar y el estado psicológico de la fruta, lo que hace a la red neuronal, con su capacidad de cambio, una solución eficiente.

De otra parte, Brosnan y Sun (40) apuntan un estudio de red neuronal para productos alimenticios, en el que se tuvieron en cuenta características como el tamaño, la textura, el color y el grado de aparición de magulladuras, para determinar el nivel de aceptabilidad de compra, concluyendo que el análisis de imágenes, utilizando visión computa- 
cional, se convierte potencialmente en una técnica importante en la industria alimenticia.

MLP proponen evaluar, mediante el uso de redes neuronales artificiales, el cambio en apariencia y humedad de las manzanas sometidas durante un período de tiempo al secado a temperaturas oscilantes entre $50^{\circ} \mathrm{C}$ y $70^{\circ} \mathrm{C}$; obtienen que el modelo MLP ANN aproxima satisfactoriamente las variaciones en el color y la humedad en las manzanas con coeficiente de correlación mayor a 0.92.

De esta manera, las redes neuronales también han permitido desarrollar nuevas formas de resolver algunos problemas que, de por sí, ya contaban con una forma de resolverse, pero que le aportan a la disminución de tiempo, mayor efectividad y fiabilidad en las respuestas.

C. Predicción. La otra aplicación muy común de las RNA es la predicción de las cosechas. Teniendo en cuenta algunos patrones y datos ya conocidos, las RNA pueden determinar las fechas de cosecha para casos extremos. Los productos trabajados han sido los pimientos dulces, los tomates, los olivos, las manzanas y la lechuga. Para los pimientos dulces, Lin y Hill (42) propusieron modelar la coloración de cada fruta individualmente, y predecir la fecha de cosecha monitoreando la intensidad de colores rojos, verdes y azules del desarrollo del pimiento, vía procesamiento de imágenes digitales, llegando a la conclusión de que utilizando redes neuronales se obtienen mejores predicciones de los días de cosecha de los pimientos dulces, en comparación con los modelos de regresión lineal múltiple.

Para el caso de la manzana, Ochoa-Martínez y Ayala-Aponte (43)immersion time, surface area, solution to fruit mass ratio and agitation level desarrollaron una red neuronal artificial para predecir la pérdida de agua y la ganancia de solido en la deshidratación osmática en los cilindros de la manzana; el modelo correlaciona seis variables de procesamiento (temperatura y concentración de la solución osmática, tiempo de inmersión, área de la superficie, ratio de masa de la solución a la fruta y nivel de agitación).
Igualmente, Ehret et al. (44) proponen relacionar la aparición de grietas en los tomates y pimientos con las condiciones de invernadero y cultivo, para predecir cuándo ocurrirá el agrietamiento durante su proceso de crecimiento en invernaderos comerciales. La construcción de la red neuronal feed-forward es un proceso de prueba y error y se hizo con el software NeuralWorks Predict v3.13. Se produjeron como resultado varios modelos de redes neuronales, uno para los tomates y otro para los pimientos, que realizan su función adecuadamente con los datos recogidos.

Por su parte, Lin y Hill (45) buscaron predecir los rendimientos semanales de pimientos dulces en un invernadero, teniendo en cuenta datos comerciales y los datos obtenidos durante 5 años, debido a que los rendimientos de los pimientos dulces son muy irregulares y no permiten conocer cuándo será la fecha de cosecha. El artículo concluyó que la red neuronal es apropiada y responde adecuadamente con los datos introducidos para las primeras semanas, pero tiene problemas con la predicción para la tercera y cuarta semana, debido a que no correspondían a los patrones usuales.

Por otro lado, en el caso de la lechuga, Lin y Block (46) propusieron predecir la vida útil de la lechuga bajo fluctuaciones de temperatura después de su cosecha. Los signos que se tienen presentes para el análisis de la lechuga son el decaimiento y el amarillamiento después del ciclo de vida. El artículo concluyó que los modelos NN tienen gran potencial para el control de calidad de la cadena fría y la predicción de vida útil.

Identificar características como la humedad en la fruta puede facilitar la toma de decisiones sobre la cosecha; Khoshhal et al. (47) aplican RNA para predecir el ratio de humedad en el proceso de secado de la manzana; tienen en consideración como entradas para la RNA la velocidad y la temperatura del aire, el espesor de las rebanadas y el tiempo de secado.

A su vez, el entrenamiento por diferentes algoritmos de aprendizaje puede o no proporcionar los resultados deseados; Meisami-asl y Rafiee (48) usan una RNA con retroalimentación entrenada 
con algoritmos Levenverg-Marquard y momentum, concluyendo que el primero proporciona mejores resultado que el segundo para predecir la masa de la manzana con tres parámetros de entrada: volumen, densidad y diámetro principal.

El color no es el único atributo característico para identificar el cambio en las frutas; Zarifneshat et al. (49) proponen el uso de la RNA como alternativa para la predicción del volumen de las maguIladuras en las manzanas Golden Delicious; la red neuronal fue entrenada con los algoritmos de BB (basic back propagation) y BDLRF (backpropagation with declining learning-rate factor algorithm), concluyendo que el segundo método (BDLRF) obtiene mejores resultados.

En el caso de los olivos, y con el fin de lograr una óptima recolección de los frutos, Gatica et al. (50) proponen el uso de redes neuronales artificiales para el reconocimiento de la fruta de los olivos, analizando las imágenes y evaluando el tamaño y el peso, características normalmente tenidas en cuenta en una inspecciona manual.

Con el fin de predecir el peso como atributo relacionado directo a la calidad de los manojos de los bananos, Soares et al. (51) describen un protocolo usando RNA, mostrando una efectividad en la predicción muy alta.

Garantizar la buena siembra asegura, de algún modo, una buena cosecha; es así como Zúñiga et al. (52) proponen analizar las semillas de las uvas usando metodologías de reconocimiento de patrones y clasificarlas en inmaduras, maduras y sobremaduradas, a través de una red neuronal con aprendizaje supervisado, con el fin de determinar el tiempo para cosechar.

\section{Conclusiones}

La revisión de la literatura reveló que hay gran variedad de investigaciones dirigidas a la aplicación de redes neuronales artificiales y de visión artificial a la clasificación, inspección y predicción de los cambios físicos de las frutas y hortalizas. Debido a la capacidad de las redes neuronales para aprender patrones de un conjunto de datos no lineales y con presencia de ruido, en los estudios mencionados en este artículo se presentaron una amalgama de arquitecturas, topologías y algoritmos de entrenamiento de redes neuronales como alternativas a la clasificación manual, los cuales ofrecieron, en muchos casos, una solución eficiente y efectiva.

Se identificó que, en cada investigación considerada, los autores se centraron en la importancia de los datos de entrada, así como en su previo tratamiento, en la arquitectura y topología de la red y en la selección de los algoritmos de entrenamiento, asegurando que estos ítems proporcionan mejor o peor clasificación, según el manejo dado. Dentro de los estudios descritos, la red neuronal más utilizada fue la Backpropagation (BPNN), seguida de la de Boltzman (BPN) y la red Probabilística (PNN).

\section{Referencias}

(1) Kung SY. Digital neural networks. Prentice Hall, 1993.

(2) Hassoun MH. Threshold Gates, in Fundamentals of Artificial Neural Networks. MIT Press, 1995.

(3) Bishop CM. Statical Pattern Recognition, in Neural Networks for Pattern Recognition. Oxford University Press, 1995.

(4) Chen G. and Dong X., From Chaos to Order: Methodologies, Perspectives, and Applications. World Scientific, 1998. DOI: http://dx. doi.org/10.1142/3033.

(5) Fausett LV. Fundamentals of Neural Networks: Architectures, Algorithms, and Applications. Prentice-Hall, 1994, pp. 1-7.

(6) Lin CT, Lin CT and Lee CSG. Neural fuzzy systems: a neuro-fuzzy synergism to intelligent systems. Prentice Hall PTR, 1996.

(7) Rosenblatt F and Laboratory CA. The perceptron: a theory of statistical separability in cognitive systems (Project Para). Cornell Aeronautical Laboratory, 1958. 
(8) Werbos PJ. The Roots of Backpropagation: From Ordered Derivatives to Neural Networks and Political Forecasting. J. Wiley \& Sons, 1994.

(9) González JRH, Lez JRHG, Coaut MHVJ and Hernando VJM. Redes neuronales artificiales: Fundamentos, modelos y aplicaciones. Addison-Wesley Iberoamericana, 1995.

(10) Serrano AJ, Soria E. and Martín JD. Redes Neuronales - OCW de la Universitat de Valencia, 2010. [En línea]. Disponible en: http://ocw. uv.es/ingenieria-y-arquitectura/1-2/Course listing.

(11) Sucar LE and Gómez G. Procesamiento del color, in Visión Computacional. p. 60.

(12) Mejía Vilet JR. Procesamiento del color, en Procesamiento Digital de Imágenes, p. 64.

(13) Parker JR. Edge-Detection Techniques, in Algorithms for Image Processing and Computer Vision. John Wiley \& Sons, 2010, p. 39.

(14) Ben-Hanan U., Peleg K and Gutman P. Classification of fruits by a boltzmann perceptron neural network, Automatica, 1992; 28 (5): 961968. DOI: http://dx.doi.org/10.1016/00051098(92)90148-9.

(15) Yang Q. Classification of apple surface features using machine vision and neural networks. Computers and Electronics in Agriculture, 1993; 9 (1): 1-12. DOI: http://dx.doi. org/10.1016/0168-1699(93)90025-V.

(16) Nakano K. Application of neural networks to the color grading of apples. Computers and Electronics in Agriculture, 1997; 18 (2-3): 105116. DOI: http://dx.doi.org/10.1016/S01681699(97)00023-9.

(17) Morimoto T. and Hashimoto Y. An intelligent control for greenhouse automation, oriented by the concepts of SPA and SFA - an application to a post-harvest process. Computers and Electronics in Agriculture, 2000; 29 (1-2): 179-
194. DOI: http://dx.doi.org/10.1016/S01681699(00)00133-2.

(18) Effendi Z., Ramli R., Ghani JA and Rahman MNA. A Back Propagation Neural Networks for Grading Jatropha curcas Fruits Maturitiy. American Journal of Applied Sciences, 2010; 7 (3): 390-394. DOI: http://dx.doi.org/10.3844/ ajassp.2010.390.394.

(19) Mustafa NBA, Arumugam K., Ahmed SK and Sharrif ZAM. Classification of fruits using Probabilistic Neural Networks - Improvement using color features. IEEE, 2011, pp. 264269. DOI: http://dx.doi.org/10.1109/TENCON.2011.6129105.

(20) Jayas DS, Paliwal J and Visen NS. Multi-layer Neural Networks for Image Analysis of Agricultural Products. Journal of Agricultural Engineering Research, 2000; 77 (2): 119-128. DOI: http://dx.doi.org/10.1006/jaer.2000.0559.

(21) Leemans V., Magein H. and Destain MF. Fruit Grading according to their External Quality using Machine Vision. Biosystems Engineering, 2002; 83 (4): 397-404. DOI: http://dx. doi.org/10.1006/bioe.2002.0131.

(22) Kavdır I. and Guyer DE. Comparison of Artificial Neural Networks and Statistical Classifiers in Apple Sorting using Textural Features. Biosyst. Eng., 2004; 89 (3): 331-344. DOI: $\quad$ http://dx.doi.org/10.1016/j.biosystemseng.2004.08.008.

(23) Xiaobo Z., Jiewen Z and Yanxiao L. Apple color grading based on organization feature parameters. Pattern Recognit. Lett, 2007; 28 (15): 2046-2053. DOI: http://dx.doi.org/10.1016/j. patrec.2007.06.001.

(24) Kondo N., Ahmad U., Monta M. and Murase $\mathrm{H}$. Machine vision based quality evaluation of lyokan orange fruit using neural networks. Computers and Electronics in Agriculture, 2000; 29 (1-2): 135-147. 
(25) Morimoto T., Takeuchi T., Miyata H and Hashimoto Y. Pattern recognition of fruit shape based on the concept of chaos and neural networks. Computers of Electronics in Agriculture, 2000; 26 (2): 171-186. DOI: http://dx.doi. org/10.1016/S0168-1699(00)00070-3.

(26) Hobani Al, Thottam AM and Ahmed KAM. Development of a Neural Network Classifier for Date Fruit Varieties Using Some Physical Attributes. Agric Res Cent. King Saud Univ, 2003; 126: 5-18.

(27) Ashok V. and Vinod DS. Automatic quality evaluation of fruits using Probabilistic Neural Network approach. IEEE, 2014; 308-311.

(28) Simões AS, Reali Costa A., Hirakawa AR and Saraiva AM. Applying neural networks to automated visual fruit sorting, in World Congress of Computer in Agriculture WCCA, 2001; 1-7.

(29) Ji H. and Yuan J. The Application Study of Apple Color Grading by Particle Swarm Optimization Neural Networks. IEEE, 2006; 1: 2651-2654. DOI: http://dx.doi.org/10.1109/ WCICA.2006.1712843.

(30) Unay D. and Gosselin B. Automatic defect segmentation of 'Jonagold' apples on multi-spectral images: A comparative study. Postharvest Biol. Technol., 2006; 42 (3): 271-279. DOI: http://dx.doi.org/10.1016/j.postharvbio.2006.06.010.

(31) Zhang Y., Wang S., Ji G and Phillips P. Fruit classification using computer vision and feedforward neural network. Journal Food Engineering, 2014; 143: 167-177. DOI: http://dx. doi.org/10.1016/j.jfoodeng.2014.07.001.

(32) Brosnan T. and Sun DW. Inspection and grading of agricultural and food products by computer vision systems-a review. Comput. Electron. Agric., 2002; 36 (2-3): 193213. DOI: http://dx.doi.org/10.1016/S01681699(02)00101-1.
(33) Fernández Andrés JC, Suardíaz Muro J., Navarro Lorente PJ, Toledo Moreo A., Jiménez Buendía M., Ortiz Zaragoza FJ and Iborra García AJ. Uso de Redes Neuronales para el Análisis de Formas Naturales, 2002; 1-4.

(34) Kavdir I. and Guyer DE. Apple sorting using artificial neural networks and spectral imaging. Trans.-Am. Soc. Agric. Eng., 2002; 45 (6): 1995-2006.

(35) Shahin MA, Tollner EW, McClendon RW and Arabnia HR. Apple classification based on surface bruises using image processing and neural networks. Trans.-Am. Soc. Agric. Eng., 2002; 45 (5): 1619-1628.

(36) Li and Zhu W. Apple Grading Method Based on Features Fusion of Size, Shape and Color. Procedia Engineering, 2011; 15: 28852891. DOI: http://dx.doi.org/10.1016/j. proeng.2011.08.543.

(37) Kurtulmus F., Lee W. and Vardar A. Immature peach detection in colour images acquired in natural illumination conditions using statistical classifiers and neural network. Precision Agric, 2014; 15 (1): 57-79. DOI: http://dx.doi. org/10.1007/s11119-013-9323-8.

(38) Wang S., Zhang Y., Ji G., Yang J., Wu J and Wei L. Fruit Classification by Wavelet-Entropy and Feedforward Neural Network Trained by Fitness-Scaled Chaotic ABC and Biogeography-Based Optimization. Entropy, 2015; 17 (8): 5711-5728. DOI: http://dx.doi. org/10.3390/e17085711.

(39) Morimoto T., Purwanto W., Suzuki J. and Hashimoto Y. Optimization of heat treatment for fruit during storage using neural networks and genetic algorithms. Computers of Electronics in Agriculture, 1997; 19 (1): 87-101. DOI: http:// dx.doi.org/10.1016/S0168-1699(97)00037-9.

(40) Brosnan T. and Sun DW. Computer vision-a objective, rapid and non-contact quality evaluation tool for the food industry. Journal Food 
Engineering, 2004; 61 (1): 3-16. DOI: http:// dx.doi.org/10.1016/S0260-8774(03)00182-1.

(41) Hossein Nadian M., Rajiee S., Aghbashlo M., Hosseinpour S and Saeid Mohtasebi S. Continuous real-time monitoring and neural network modeling of apple slices color changes during hot air drying, Food and Bioproducts Processing. Trans. Inst. Chem. Eng. Part C., 2015; 94: 263-274. DOI: http://dx.doi.org/10.1016/j.fbp.2014.03.005.

(42) Lin WC and Hill BD. Neural network modeIling of fruit colour and crop variables to predict harvest dates of greenhouse-grown sweet peppers. Foreword, 2007; 87 (1): 137-143. DOI: http://dx.doi.org/10.4141/p05-231.

(43) Ochoa-Martínez $\mathrm{Cl}$ and Ayala-Aponte AA. Prediction of mass transfer kinetics during osmotic dehydration of apples using neural networks. LWT - Food Sci. Technol., 2007; 40 (4): 638-645.

(44) Ehret DL, Hill BD, Raworth DA and Estergaard B. Artificial neural network modelling to predict cuticle cracking in greenhouse peppers and tomatoes. Computers of Electronics in Agriculture, 2008; 61 (2): 108-116. DOI: http:// dx.doi.org/10.1016/j.compag.2007.09.011.

(45) Lin WC and Hill BD. Neural network modeIling to predict weekly yields of sweet peppers in a commercial greenhouse. Can. J. Plant Sci., 2008; 88 (3): 531-536. DOI: http://dx.doi. org/10.4141/cjps07165.

(46) Lin WC and Block GS. Neural Network Modeling to Predict Shelf Life of Greenhouse Lettuce. Algorithms, 2009; 2 (2): 623-637. DOI: http://dx.doi.org/10.3390/a2020623.

(47) Khoshhal A., Dakhel AA, Etemadi A and Zereshki S. Artificial Neural Network Modeling of Apple Drying Process. Journal Food Process Engineering, 2010; 33: 298313. DOI: http://dx.doi.org/10.1111/j.17454530.2009.00435.x.
(48) Meisami-asI E and Rafiee S. Modeling of physical properties of apple slices (Golab variety) using artificial neural networks. Agric. Eng. Int. CIGR J., 2012; 14 (3): 175-178.

(49) Zarifneshat S., Rohani A., Ghassemzadeh HR, Sadeghi M., Ahmadi E and Zarifneshat M. Predictions of apple bruise volume using artificial neural network. Computers of Electronics in Agriculture, 2012; 82: 75-86. DOI: http://dx. doi.org/10.1016/j.compag.2011.12.015.

(50) Gatica G., Best S., Ceroni J. and Lefranc G. Olive Fruits Recognition Using Neural Networks. Procedia Computer Science, 2013; 17: 412-419. DOI: http://dx.doi.org/10.1016/j. procs.2013.05.053.

(51) Soares JD, Pasqual M., Lacerda WS, Silva SO and Donato SLR. Utilization of artificial neural networks in the prediction of the bunches' weight in banana plants. Scientia Horticulturae, 2013; 155: 24-29. DOI: http://dx.doi.org/10.1016/j.scienta.2013.01.026.

(52) Zuñiga A., Mora M., Oyarce M. and Fredes C. Grape maturity estimation based on seed images and neural networks. Engineering Applications of Artificial Intelligence, 2014; 35: 95-104. DOI: http://dx.doi.org/10.1016/j. engappai.2014.06.007.

(53) Evin D., Hadad A., Martina M. and Drozdowicz B. Predicción de estados de hipotensión empleando modelos ocultos de Markov. Fac. Ing., 2011; 20 (30): 55-63. DOI: http:// dx.doi.org/10.19053/01211129.1418.

(54) Fuertes W., Rodas F. and Toscano D. Evaluación de ataques UDP Flood utilizando escenarios virtuales como plataforma experimental. Fac. Ing., 2011; 20 (31): 37-53. DOI: http:// dx.doi.org/10.19053/01211129.1422. 